\title{
Mark Schweda, Larissa Pfaller, Kai Brauer, Frank Adloff and Silke Schicktanz (eds.) (2017). Planning Later Life. Bioethics and Public Health in Ageing Societies. Abingdon/ New York: Taylor \& Francis [Routledge Advances in Health and Social Policy], 263 pp. ISBN 9781472481320 (hardback)
}

\author{
REVIEWED by MALTE VÖLK*
}

The conceptual linchpin of this volume, as it is laid out in the introduction and in the editor's further contributions, is the juxtaposition of contemporary Ageing Studies and a perspective on human life that sees as given a somehow predetermined life course. The book's title, Planning Later Life, is to be understood in such a context, embedded in the broader question: How do different cultural fields - medicine being one of the most important ones - define what is appropriate for the aged? Ethical and philosophical considerations as well as case studies from different parts of the world try to determine what influence these cultural images have on the individual and societal planning of later life. In order to implement its complex and ambitious combination of individual and societal aspects of ageing societies, the book brings together contributions from distinguished scholars, 
International Journal of Ageing and Later Life

often building on the results of many years of research - conducted in different fields of science and humanities, allowing for the combination of philosophical, bio-ethical, anthropological and sociological viewpoints and providing fruitful insights into a central issue of human development in the light of growing life expectancies.

The book consists of three parts. The first part with contributions from Mark Schweda, Thomas Rentsch, François Höpflinger and Paul Higgs/ Chris Gilleard looks at "Conceptions of ageing and old age" in a rather broad scope. With a main emphasis on philosophical and sociological perspectives, the changes in those conceptions are identified and reflected, especially in terms of notions of a naturally determined life course that not only create images of age-appropriate behaviour but can lead to grave consequences in the area of public health. The second part, entitled "Perspectives and problems of old age in the context of medicine and healthcare," combines contributions from Andreas Kruse, Perla Werner/ Silke Schicktanz, Hsiu-I Yang, Søren Holm and Nancy S. Jecker. Here, a variety of specific issues are discussed, ranging from old age in modern society over ethical questions alongside the occurrence of dementia and end-of-life decisions to bio-technological life extensions. The last part of the book, with contributions from Kai Brauer, Ralf J. Jox, S. Jay Olshansky, Larissa Pfaller/Frank Adloff, Silke Schicktanz, Ruud ter Meulen and Stephen Katz/Peter J. Whitehouse, focuses on the current modes of strategies of planning later life, analysing the efforts of countering its individual and societal contingency.

What these three parts have in common is a critical view on normative and still widespread conceptions that tend to repress and devalue old age. One reaction towards these could be to change the perspective and focus on the question what societies can learn from the phenomenon of ageing. The answer, according to Rentsch, is that the fact and meaning of ageing let society gain valuable reflections on modesty. The answer is even outperformed by Katz/Whitehouse: "Globally, rather than being a mega-problem, ageing [...] can be inverted to become a mega-solution because the transfer of human repositories of experience, stories and wisdom is one way to deepen resistance to social instability" (Katz/Whitehouse 2017: 250).

However, the idea of turning the tables should not be confused with another normative conception that would aim at "replacing one biased 
stereotype of old age with another, thus merely reversing the value system that informs ethical discourse" (Schweda 2017: 26). Rejecting onedimensional ethical viewpoints, Schweda demonstrates how different sets of meaning and value are attributed to different stages of life through internalised stereotypes. Such implicit pictures of a natural life course can become dangerously explicit when medical treatment is needed: studies show that the age of individuals does in fact influence decisions in a way that indicates costly treatments seem to be seen as less worthwhile when older people would benefit from them.

In a similar vein, Andreas Kruse in his definition of seven factors that should constitute an "age-friendly culture" demands as a central point: "Decisive for healthcare provision is the diagnosis alone, made by an expert, not the age of the individual concerned" (Kruse 2017: 87). This manifesto for duly balanced intergenerational relationships, that also includes aspects like workplace environments and societal communication, is derived from an analysis of the general nature of old age today that is shaped by the observation of two human strains of ageing: While the perils of the physical process of ageing need not be denied, they are getting too much attention and divert the view on "the potential wealth that can unfold in the ageing process" (Kruse 2017: 75) in terms of mental experience. But this is not to be understood as a sharp distinction between a positive (active, participative) "third age" and a "fourth age," which might not be worth living anymore. Conceptions like these are contested by many of the contributions gathered here, and Kruse also argues that even in situations of extreme vulnerability like severe dementia, there can still be individual emotional experience and meaningful interactions, as evidenced by empirical research. Thus, the quality of life cannot be measured in universal scales. The same problem seems to apply for scales and measurements of decision-making competence in the process of cognitive deterioration: an area, where still "not enough attention is being paid to ethical issues" (Werner/Schicktanz 2017: 97), as Perla Werner and Silke Schicktanz point out.

Hsiu-I Yang raises a topic that extends ethical questions like these towards life-sustaining medical technology. Her thoughts are restricted to cases without chances of healing or even the regaining of consciousness - but still, as she proposes to grant individuals above the age of 80 years a "peaceful death" (Yang 2017: 110) and wishes to establish "a 
International Journal of Ageing and Later Life

mature culture of death, in which old people can live longer and healthier and die quicker and fitter" (Yang 2017: 113), her line of argumentation is not free from the notion of a natural life course with fixed stages: how else could having lived a "full life" be understood as having turned 80 , as she defines it?

The contributions presented in this book display the current state of discussions in Ageing Studies, and with their specific focus on the expected future scenarios and planning processes, they bring out the dynamics of ageing societies in an impressive way. It has to be mentioned, though, that the strength of the volume does not lie in the interaction of the articles. Their heterogeneity can be obtrusive at times. Still, the benefits of the diverse range of topics and disciplines outweigh this point, which is also a marker for the necessity of ongoing discussions in the dynamic field of Ageing Studies. For this, Planning Later Life provides some excellent foundations that can prove to be important for scholars from various disciplines.

\section{References}

Katz, S. \& Whitehouse, P. J. (2017). Legacies, generations, and ageing futures: the ethics of intergenerativity. In M. Schweda, L. Pfaller, K. Brauer, F. Adloff \& S. Schicktanz (eds.), Planning Later Life. Bioethics and Public Health in Ageing Societies (pp. 240-253). Abingdon/New York: Taylor \& Francis.

Kruse, A. (2017). Old age, potentials and vulnerability. In M. Schweda, L. Pfaller, K. Brauer, F. Adloff \& S. Schicktanz (eds.), Planning Later Life. Bioethics and Public Health in Ageing Societies (pp. 75-88). Abingdon/New York: Taylor \& Francis.

Schweda, M., (2017). "A season to everything"? Considering life-course perspectives in bioethical and public-health discussions on ageing. In M. Schweda, L. Pfaller, K. Brauer, F. Adloff \& S. Schicktanz (eds.), Planning Later Life. Bioethics and Public Health in Ageing Societies (pp. 11-30). Abingdon/New York: Taylor \& Francis.

Werner, P. \& Schicktanz, S. (2017). Competence and cognitive detoriation: are we paying enough attention to ethical issues? In M. Schweda, L. Pfaller, K. Brauer, F. Adloff \& S. Schicktanz (eds.), Planning Later Life. 
Bioethics and Public Health in Ageing Societies (pp. 89-103). Abingdon/ New York: Taylor \& Francis.

Yang, Hsiu-I (2017). Opt in or opt out? Rethinking the provision of life-sustaining medical technology to the 'old old'. In M. Schweda, L. Pfaller, K. Brauer, F. Adloff \& S. Schicktanz (eds.), Planning Later Life. Bioethics and Public Health in Ageing Societies (pp. 104-118). Abingdon/New York: Taylor \& Francis. 
